\title{
Ion acoustic quasi-soliton in an electron-positron-ion plasma with superthermal electrons and positrons
}

https://doi.org/10.1515/phys-2018-0073

Received February 23, 2018; accepted May 21, 2018

\begin{abstract}
In this work, we are concerned with the ion acoustic quasi-soliton in an electron-positron-ion plasma with superthermal electrons and positrons. By using the reductive perturbation method, the Korteweg-de Vries equation is derived from the governing equations of ion acoustic waves. An interesting soliton-cnoidal wave solution of the Korteweg-de Vries equation and its quasisoliton behaviour are presented. The influence of electron superthermality, positron superthermality and positron concentration ratio on characteristics of the quasi-soliton is confirmed to be significant.
\end{abstract}

Keywords: Ion acoustic wave, electron-positron-ion plasma, KdV equation, quasi-soliton behaviour

PACS: 52.35.Fp, 52.35.Mw, 52.35.Sb, 52.35.Tc

\section{Introduction}

Ion acoustic solitary waves, a fundamental mode in nonlinear plasma environments, have been a subject of extensive research for several decades [1-5]. There are two main approaches to study ion-acoustic solitary waves, namely, the reductive perturbation method and the pseudopotential theory. For small-amplitude excitation, the re-

\footnotetext{
Jianyong Wang: Department of Mathematics and Physics, Quzhou University, Quzhou 324000, China

Ying Zeng: Department of Mathematics and Physics, Quzhou University, Quzhou 324000, China

^Corresponding Author: Zufeng Liang: Department of Physics, Hangzhou Normal University, Hangzhou, 310036, P. R. China, E-mail: liangzufeng@163.com

Yani Xu: College of Teacher Education, Quzhou University, Quzhou 324000, China

Yuanxiang Zhang: Key Laboratory of Air-driven Equipment Technology of Zhejiang Province, Quzhou University, Quzhou 324000, China
}

ductive perturbation technique is applicable. As is widely known, the Korteweg-de Vries (KdV) equation and its generalizations are very useful approximation to the full set of fluid equations describing ion acoustic waves [1]. Recently, Saha et al. studied bifurcation and quasi-periodic behaviours of ion acoustic waves in a magnetized electronion (e-i) plasma with nonthermal electrons featuring Cairns-Tsallis distribution [6]. The Kadomtsev-Petviashvili equation equation is derived by employing the reductive perturbation method, and used to analyze the existence of solitary wave solutions and periodic traveling wave solutions. In addition to solitary waves, the study of cnoidal waves in field free or magnetized plasmas is another topic of great interest due to its importance in the nonlinear transport processes in plasmas [7-12]. Recently, Saha and Chatterjee studied ion acoustic periodic waves in an unmagnetized plasma with superthermal electrons [12]. By applying a non-perturbative approach, they obtained a planar dynamical system and showed that plasma parameters significantly affect the characteristics of periodic wave structures. Besides, the interaction between solitons, such as the head-on collisions of ion acoustic waves, have been intensively studied by means of the extended Poincaré-Lighthill-Kuo perturbation method [13-18].

However, interactions among different types of nonlinear waves in e-i plasmas as well as electron-positronion (e-p-i) plasmas are rarely considered. Recently, Keane et al. [20] considered the propagation of Alfvén envelope solitons in a quantum plasma. It is found that the timeindependent Alfvén density soliton possesses a quasisoliton structure with the Gauss peak surrounded by small amplitude sinusoidal waves. Furthermore, it was numerically confirmed that the shape of the Gauss peak remains the same in space-time evolution. This interesting numerical phenomena reveals that the interaction between the soliton core and its surrounded oscillations is elastic. Consequently, it seems significant if one can establish an analytical solution to explain such type of wave interactions. Fortunately, analytic solutions, describing interactions between solitons and other nonlinear excitations, have been 
obtained by combining the symmetry reduction method with the Darboux transformation or Bäcklund transformation related nonlocal symmetries [21, 22]. Then, the consistent Riccati expansion method has been proposed to find the interacting wave solutions for many integrable systems [23-25]. It turns out that the KdV, nonlinear Schrödinger, and sine-Gordon equations possess different types of interacting wave solutions, such as nanopteron solution [26] or topological soliton on a cnoidal wave background [27].

In this paper, we are concerned with the propagation of ion acoustic quasi-soliton in an e-p-i plasma with superthermal electrons and positrons. In the next section, the KdV equation, describing the propagation of nonlinear ion acoustic waves in an e-p-i plasma, is derived by applying the reductive perturbation method. In addition, exact soliton-cnoidal wave solution of the KdV equation and its quasi-soliton behaviour are presented. The influence of plasma parameters on characteristics of the quasi-soliton is presented in the third section. The last section is a discussion.

\section{$2 \mathrm{KdV}$ equation and its quasi-soliton solution}

We are interested in studying ion acoustic quasi-soliton in a fully ionized, collisionless unmagnetized plasma, whose components are cold ions, superthermal electrons and superthermal positrons. Adopting a one dimensional fluid formulation, the dynamics of the cold inertial ion component, ignoring the thermal pressure effect, is governed by the following dimensionless set of fluid equations

$$
\begin{gathered}
\frac{\partial n_{i}}{\partial t}+\frac{\partial\left(n_{i} u_{i}\right)}{\partial x}=0, \\
\frac{\partial u_{i}}{\partial t}+u_{i} \frac{\partial u_{i}}{\partial x}=-\frac{\partial \phi}{\partial x}, \\
\frac{\partial^{2} \phi}{\partial x^{2}}=n_{e}-p n_{p}-(1-p) n_{i},
\end{gathered}
$$

where $u_{i}$ is the dimensionless ion hydrodynamic velocity normalized by the ion acoustic speed $C_{s}=\left(T e / m_{i}\right)^{(1 / 2)}$ with the electron temperature $T_{e}$ and the rest ion mass $m_{i} . n_{i}, n_{e}$, and $n_{p}$ are ion, positron and electron number densities normalized by their equilibrium values $n_{i 0}, n_{e 0}$, and $n_{p 0}$, respectively. Under the slow ion acoustic wave time scale, annihilation between electrons and positrons is negligible. Thus, we assume that the number density of electrons and positrons satisfies the following distribution functions

$$
n_{e}=\left(1-\frac{\phi}{\kappa_{e}-\frac{3}{2}}\right)^{-\kappa_{e}+\frac{1}{2}}, n_{p}=\left(1+\frac{\sigma \phi}{\kappa_{p}-\frac{3}{2}}\right)^{-\kappa_{p}+\frac{1}{2}},
$$

with $\sigma=T_{e} / T_{p}$ being the electron to positron temperature ratio, $\phi$ is the ion acoustic wave potential normalized by $T_{e} / e$, and $p=n_{p 0} / n_{e 0}$ is the fractional concentration of positrons with respect to electrons in the equilibrium state. It is noted that time variable $t$ is normalized ion plasma period $\omega_{p i}^{-1}=\sqrt{m_{i} /\left(4 \pi n_{e 0} e^{2}\right)}$, and space variable $x$ is normalized by the Debye length $\lambda_{D e}=\sqrt{T_{e} /\left(4 \pi n_{e 0} e^{2}\right)}$.

Under the weak perturbation assumption with $e \phi / T_{e} \ll 1$, we obtain the following approximation of the Poisson's equation by substituting the distribution functions of electrons and positrons (4) into equation (3)

$$
\frac{\partial^{2} \phi}{\partial x^{2}} \simeq(1-p)\left(1-n_{i}\right)+a_{\kappa} \phi+\frac{1}{2} b_{\kappa} \phi^{2},
$$

where

$$
\begin{gathered}
a_{\kappa}=\frac{2 \kappa_{e}-1}{2 \kappa_{e}-3}+\frac{\left(2 \kappa_{p}-1\right) p \sigma}{2 \kappa_{p}-3}, \\
b_{\kappa}=\frac{4 \kappa_{e}^{2}-1}{\left(2 \kappa_{e}-3\right)^{2}}-\frac{\left(4 \kappa_{p}^{2}-1\right) p \sigma^{2}}{\left(2 \kappa_{p}-3\right)^{2}} .
\end{gathered}
$$

In order to investigate the nonlinear excitations of small-amplitude ion acoustic waves, we employ the reductive perturbation technique. A set of stretched variables are introduced as

$$
\xi=\epsilon^{1 / 2}(x-V t), \quad \tau=\epsilon^{3 / 2} t,
$$

with $\epsilon$ is a small parameter lies in the range $0<\epsilon<1$, and $V$ is the normalized phase speed to be determined later. The perturbed quantities are expanded about their equilibrium states as

$$
\begin{aligned}
& n_{i}=1+\epsilon n_{1}+\epsilon^{2} n_{2}+\cdots, \\
& \phi=\epsilon \phi_{1}+\epsilon^{2} \phi_{2}+\cdots, \\
& u_{i}=\epsilon u_{1}+\epsilon^{2} u_{2}+\cdots
\end{aligned}
$$

Substituting equations (8)-(11) into equations (1)-(3) and setting the coefficients of different powers of $\epsilon$ to zero , one finds a sequence of differential equations. From the order of $\epsilon^{3 / 2}$, we obtain the compatibility conditions about $n_{1}, u_{1}$ and $\phi_{1}$, which can be solved as

$$
n_{1}=\frac{a_{\kappa}}{1-p} \phi_{1}, u_{1}=\frac{1}{V} \phi_{1}, V=\sqrt{\frac{1-p}{a_{\kappa}}} .
$$

The second order terms in $\epsilon$ yield a further set of compatibility conditons. Together with the known results (12), we have

$$
n_{2}=\frac{b_{\kappa}}{2(1-p)} \phi_{1}^{2}-\frac{1}{1-p} \frac{\partial^{2} \phi_{1}}{\partial \xi^{2}}+\frac{a_{\kappa}}{1-p} \phi_{2},
$$


and

$$
u_{2}=\frac{1}{4}\left[\frac{b_{\kappa}}{a_{\kappa} V}-\frac{a_{\kappa}}{(1-p) V}\right] \phi_{1}^{2}-\frac{1}{2 a_{\kappa} V} \frac{\partial^{2} \phi_{1}}{\partial \xi^{2}}+\frac{1}{V} \phi_{2} .
$$

Eliminating the second order quantities, we obtain the $\mathrm{KdV}$ equation

$$
\frac{\partial \phi_{1}}{\partial \tau}+A \phi_{1} \frac{\partial \phi_{1}}{\partial \xi}+B \frac{\partial^{3} \phi_{1}}{\partial \xi^{3}}=0
$$

where the nonlinear and dispersive coefficients $A$ and $B$ are given by

$$
A=\frac{3}{2 V}-\frac{b_{\kappa} V^{3}}{2(1-p)}, \quad B=\frac{V^{3}}{2(1-p)} .
$$

The soliton-cnoidal wave solution of the KdV equation as well as its quasi-soliton behaviour has been studied in detail [26], which reads

$$
\begin{aligned}
\phi_{1}= & \frac{3\left(V_{1}-V_{2}\right)}{2 A G^{2}}\left[\frac{\left(m^{2}-1+2 G\right)^{2}}{\left(m^{2}-1\right)} \tanh (w)^{2}\right. \\
& \left.-2 \delta m S\left(m^{2}-1+2 G\right) \tanh (w)+m^{2}-1\right] \\
& -\frac{\left(m^{2}+7\right) V_{1}-\left(3 m^{2}+5\right) V_{2}}{2 A\left(m^{2}-1\right)},
\end{aligned}
$$

with

$$
\begin{aligned}
& w=\frac{\xi-V_{1} \tau}{W_{1}}+c_{1} \operatorname{arctanh}\left(c_{2} S(\eta, m)\right), \\
& G=1-m^{2} S^{2}+\delta m C D, \quad \eta=\frac{\xi-V_{2} \tau}{W_{2}} \\
& S \equiv \operatorname{sn}(\eta, m), \quad C \equiv \operatorname{cn}(\eta, m), \quad D \equiv d n(\eta, m) .
\end{aligned}
$$

and the wave parameters are determined as

$$
\begin{aligned}
& W_{1}=\sqrt{\frac{8 B\left(1-m^{2}\right)}{V_{1}-V_{2}}}, \quad W_{2}=\sqrt{\frac{2 B\left(1-m^{2}\right)}{V_{1}-V_{2}}}, \\
& c_{1}=\frac{\delta}{2}, \quad c_{2}=m, \quad \delta^{2}=1 .
\end{aligned}
$$

Obviously, under the ultralimit conditon $m=0(G=1)$, $V_{1}=V$ and $V_{2}=-V$, the soliton-cnoidal wave solution (17) degenerates to the classical soliton solution of the KdV equation

$$
u=\frac{3 V}{A} \operatorname{sech}^{2}\left(\frac{x-V t}{W}\right), \quad W=\sqrt{\frac{4 B}{V}} .
$$

Consequently, the solution (17) has an interesting quasisoliton behaviour under the asymptotic condition $V_{1}=V$, $V_{2}=-V$, and $m \rightarrow 0$. It is found that the soliton core profile tends to the classical soliton of the $\mathrm{KdV}$ equation while the surrounded conidal wave becomes small amplitude sinusoidal vibrations around zero. Thus, the solution (17) can be viewed as a quasi-soliton solution for its quasisoliton behaviour.

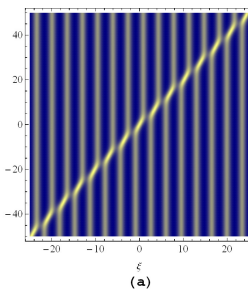

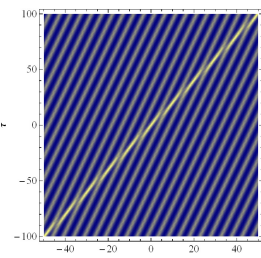

(b)

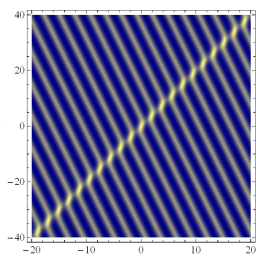

Figure 1: Density plot of the collisions between a soliton and a cnoidal wave, given by equations (17) and (18), with the plasma parameters are $\kappa_{e}=2, \kappa_{p}=5, p=0.2, \sigma=0.5$, and the wave parameters are (a) $m=1 / 3, V_{1}=0.5, V_{2}=0, \delta=1$; (b) $m=1 / 3$, $V_{1}=0.5, V_{2}=0.25, \delta=1$; and (c) $m=1 / 3, V_{1}=0.5, V_{2}=-0.25$, $\delta=1$, respectively.

\section{Parametric investigation}

In this section, we illustrate the space-time evolution of the soliton-cnoidal solution (17), and subsequently discuss the influence of relevant plasma parameters on characteristics of the quasi-soliton.

Since $V_{1}$ and $V_{2}$ are taken as the velocities of the soliton and the cnoidal wave, respectively, remain arbitrary, the soliton-cnoidal wave solution (17) can display different types of interactions. For instance, if setting $V_{2}=0$, then the cnoidal wave degenerates into a standing wave, and one can view a scenario in which a soliton moves on a standing cnoidal wave background. Fig. 1 (a) depicts a right-moving soliton colliding with a standing cnoidal wave. Fig. 1 (b) shows an elastic overtaking interaction. Fig. 1 (c) displays an elastic head-on collision between a right-going soliton and a left-going cnoidal wave. It is evident from Fig. 1 that the interaction between the soliton and every peak of the cnoidal wave is elastic with phase shifts. Interestingly, it has been shown that the collisioninduced phase shift of the cnoidal wave is half wavelength [26]

$$
\Delta_{c n}=2 W_{2} K(m)=\frac{\lambda_{c}}{2} .
$$

To demonstrate the effect of electron superthermal parameter $\kappa_{e}$, we have presented the quasi-soliton structure for fixed values of the plasma parameters, and $\kappa_{e}=$ $2,5,50$ denoting the red, green and blue curves, respectively, in Fig. 2. From the figure, one observes that the amplitude and width of the quasi-soliton flourish with increasing $\kappa_{e}$. Physically, the electron pressure provides the needed restoring force of the ion acoustic wave. Consequently, the increase in $\kappa_{e}$, which can be interpreted as an equivalent process of increasing electron pressure, makes the amplitude higher of the soliton core as well as the surrounded vibrations. On the other hand, the increase of $\kappa_{e}$ causes a larger value of the dispersion term in the $\mathrm{KdV}$ 


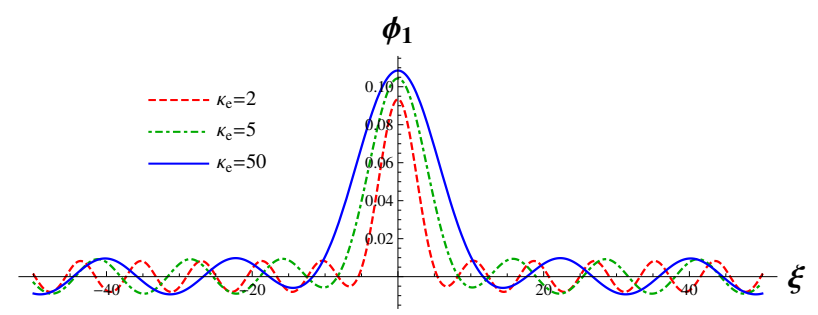

Figure 2: Variations of the ion acoustic quasi-soliton with respect to the electron superthermal parameter $\kappa_{e}$. Other plasma parameters are taken as $\kappa_{p}=50, p=0.1, \sigma=0.9$, and the wave parameters are $m=0.04, V_{1}=-V_{2}=0.05$ and $\delta=-1$.

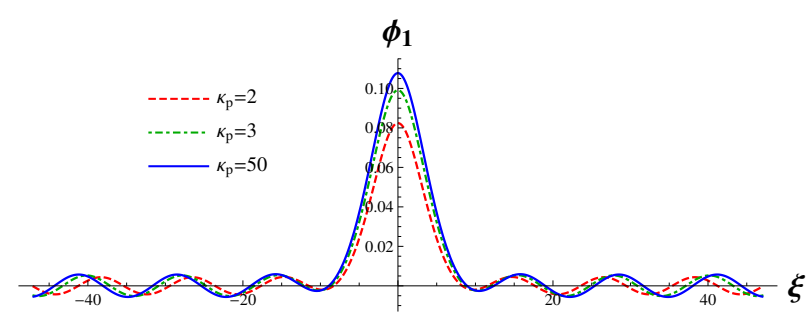

Figure 3: Variations of the ion acoustic quasi-soliton with respect to the positron superthermal parameter $\kappa_{p}$. Other parameters are $\kappa_{e}=3, p=0.1, \sigma=0.9, m=0.025, V_{1}=-V_{2}=0.05$ and $\delta=-1$.

equation, and leads to wider quasi-solitons. So increasing superthermality (smaller $\kappa_{e}$ ) leads to lower amplitude and narrower quai-solitons. It also should be noted that the amplitude and the width of the quasi-soliton will reach a maximum in the Maxwellian limit $\kappa_{e} \rightarrow \infty$. In Fig. 3, a similar effect can be observed with varying positron superthermal parameter $\kappa_{p}$.

The effect of positron-to-electron concentration ratio $p=n_{p 0} / n_{e 0}$ on characteristics of the ion acoustic quasisoliton is also investigated. It is quite evident from Fig. 4 that the amplitude as well as the width of the quasi-soliton diminishes with increasing positron concentration. As is widely known, the driving force of the ion acoustic wave is offered by the ion inertia and the increasing positron concentration $p$ implies the depopulation of ions. So in-

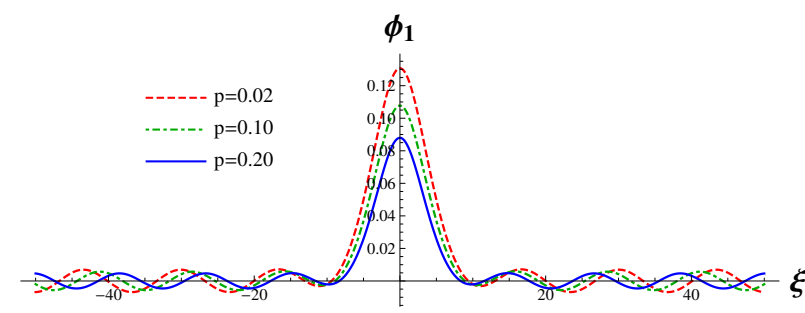

Figure 4: Variations of the ion acoustic quasi-soliton with respect to positron-to-electron concentration ratio $p$. Other plasma parameters are selected as $\kappa_{e}=3, p=0.1, \sigma=0.9$, and wave parameters are $m=0.025, V_{1}=-V_{2}=0.05$ and $\delta=1$. creasing positron concentration ratio $p$ leads to lower amplitude and narrower quai-solitons.

\section{Summary and discussion}

In this work, we are concerned with the propagation of ion acoustic quasi-soliton in an e-p-i plasma with superthermal electrons and positrons. Quasi-soliton behaviour of the soliton-cnoidal wave solution has been presented from its connection with the classical soliton solution. Numerical study shows that plasma parameters affect characteristics of the quasi-soliton significantly. It is hoped that the quasi-soliton can be observed in real nonlinear plasma environments.

Acknowledgement: The work is sponsored by National Natural Science Foundation of China (Nos. 11475052, 11605102, 11675055, 51605252).

\section{References}

[1] Washimi H., Taniuti T., Propagation of ion acoustic solitary waves of small amplitude, Phys. Rev. Lett., 1966, 17, 996-998.

[2] Lee L.C., Kan J.R., Nonlinear ion acoustic waves and solitons in a magnetized plasma, Phys. Fluids, 1981, 24, 430-433.

[3] Witt E., Lotko W., Ion acoustic solitary waves in a magnetized plasma with arbitrary electron equation of state, Phys. Fluids, 1983, 26, 2176-2185.

[4] Sultana S., Kourakis I., Saini N.S., Hellberg M.A., Oblique electrostatic excitations in a magnetized plasma in the presence of excess superthermal electrons, Phys. Plasmas, 2010, 17, 032310.

[5] Kadijani M.N., Abbasi H.,Pajouh H.H., Influence of superthermal electrons on obliquely propagating ion acoustic solitons in magnetized plasmas, Plasma Phys. Control. Fusion, 2011, 53, 025004.

[6] Saha A., Pal N., Chatterjee P., Bifurcation and Quasiperiodic Behaviors of Ion Acoustic Waves in Magnetoplasmas with Nonthermal Electrons Featuring Tsallis Distribution, Braz. J. Phys, 2015, 45, 325-333.

[7] Chawla J.K., Mishra M.K., Phys. Plasmas, ion acoustic nonlinear periodic waves in electron-positron-ion plasma, 2010, 17, 102315.

[8] Yadav L.L., Tiwari R.S., Maheshwari K.P., Sharma S.R., ion acoustic nonlinear periodic waves in a two-electrontemperature plasma, Phys. Rev. E, 1995, 52, 3045-3052.

[9] Konno K., Mitsuhashi T., Ichikawa Y.H., Propagation of ion acoustic Cnoidal Wave, J. Phys. Soc. Jpn., 1979, 46, 1907-1914.

[10] Kauschke U., Schliiter H., Forced harmonics and multimode spectra of drift waves at moderate magnetic field strengths, Plasma Phys. Contr. Fusion, 1990, 32, 1149-1175. 
[11] Ghosh U.N., Saha A., Pal N., Chatterjee P.,Dynamic structures of nonlinear ion acoustic waves in a nonextensive electronpositron-ion plasma, J. Theor. Appl. Phys. 2015, 9, 321-329

[12] Saha A., Chatterjee P., Bifurcations of ion acoustic solitary and periodic waves in an electron-positron-ion plasma through nonperturbative approach, J. Plasma Phys. 2014, 80, 553-563.

[13] Han J.N., Yang X.X., Tion acousticn D.X., Duan W.S., Head-on collision of ion acoustic solitary waves in a weakly relativistic electron-positron-ion plasma, Phys. Lett. A, 2008, 372, 48174821.

[14] El-Shamy E.F., Moslem W.M., Shukla P.K., Phys. Lett. A, Headon collision of ion acoustic solitary waves in a Thomas-Fermi plasma containing degenerate electrons and positrons, 2009, 374, 290-293.

[15] Su C.H., Mirie R.M., J. Fluid Mech., On head-on collisions between two solitary waves, 1980, 98, 509-525.

[16] Shah A., Mahmood S., Haque Q., Propagation of solitary waves in relativistic electron-positron-ion plasmas with kappa distributed electrons and positrons, Phys. Plasmas, 2011, 18, 114501.

[17] Popel S.I., Vladimirov S.V., Shukla P.K., Ion acoustic solitons in electron-positron-ion plasmas, Phys. Plasmas, 1995, 2, 716-719.

[18] Khan S.A., Masood W., Linear and nonlinear quantum ion acoustic waves in dense magnetized electron-positron-ion plasmas, Phys. Plasma, 2008, 15, 062301.
[19] Chatterjee P., Ghosh U.N., Roy K., Munion acousticndy S.V., Wong C.S.,Sahu B., Head-on collision of ion acoustic solitary waves in an electron-positron-ion plasma with superthermal electrons, Phys. Plasma, 2010, 17, 122314.

[20] Keane A.J., Mushtaq A., Wheatland M.S., Alfvén solitons in a Fermionic quantum plasma, Phys. Rev. E, 2011, 83, 066407.

[21] Cheng X.P., Lou S.Y., Chen C.L., Tang X.Y., Interactions between solitons and other nonlinear Schrödinger waves, 2014, 89, 043202.

[22] Gao X.N., Lou S.Y., Tang X.Y., Bosonization, singularity analysis, nonlocal symmetry reductions and exact solutions of supersymmetric KdV equation, JHEP, 2013, 05, 029.

[23] Lou S.Y., Consistent Riccati expansion for integrable systems, Stud. Appl. Math., 2015, 134, 372.

[24] Wang J.Y., Cheng X. P., Tang X.Y., Yang J. R., Ren B., Oblique propagation of ion acoustic soliton-cnoidal waves in a magnetized electron-positron-ion plasma with superthermal electrons, Phys. Plasma, 2014, 21, 032111.

[25] Tang X.Y., Hao X.Z., Liang Z.F., Interacting waves of DaveyStewartson III system, Comp. Math. Appl., 2017, 74, 1311-1320.

[26] Wang J.Y., Tang X.Y., Lou S.Y., Gao X.N., Jia M., Nanopteron solution of the Korteweg-de Vries equation, Europhys. Lett., 2014, 108, 20005.

[27] Tang X.Y., Liang Z.F., Wang J.Y., Nonlocal topological solitons of the sine-Gordon equation, J. Phys. A: Math. Theor., 2015, 48, 285204. 